\title{
Improving the management of spontaneous pneumothorax
}

To the Editor:

At a relatively young age, I had the misfortune to be diagnosed with spontaneous pneumothorax and go through video-assisted thoracoscopic surgery.

When a patient is admitted for spontaneous pneumothorax, the clinical staff usually mounts two types of devices on the patient: 1) an intravenous rehydration infusion set, and 2) a chest tube. When these two devices are mounted, each on a different side of the body (i.e. the intravenous rehydration infusion set is attached to the patient's right hand and the tube is attached to the left side of the patient's chest), the patient's mobility becomes limited, pain intensity may increase, and it becomes more difficult to get out of bed and walk.

Future spontaneous pneumothorax patients would feel more comfortable if both devices were mounted on the same side of the body. I believe that such a minor change in clinical practice could make a difference for many patients, and I hope that the guideline for management of spontaneous pneumothorax [1] will be updated accordingly.

@ERSpublications

Management of spontaneous pneumothorax would be improved by mounting multiple devices on the same side of the body http://ow.ly/7x7B30mrAnq

Cite this article as: Kartoun U. Improving the management of spontaneous pneumothorax. Eur Respir J 2018; 52: 1801857 [https://doi.org/10.1183/13993003.01857-2018].

\section{Uri Kartoun}

Center for Computational Health, IBM Research, Cambridge, MA, USA.

Correspondence: Uri Kartoun, Center for Computational Health, IBM Research, 75 Binney Street, Cambridge, MA 02142, USA. E-mail: uri.kartoun@ibm.com

Received: Oct 012018 | Accepted: Oct 072018

Conflict of interest: U. Kartoun received honoraria and travel funding from The American Association for the Study of Liver Diseases (October 2017).

\section{References}

1 MacDuff A, Arnold A, Harvey J. Management of spontaneous pneumothorax: British Thoracic Society Pleural Disease Guideline 2010. Thorax 2010; 65: Suppl. 2, ii18-ii31.

To the Editor:

U. Kartoun raises a point related to the improvement of future spontaneous pneumothorax guidelines based on his personal experience as a patient who underwent video-assisted thoracoscopic surgery. He suggests that inserting a peripheral venous line and a chest tube on opposite sides of the body may limit mobility and increase pain. Although it may be adventurous to judge this particular case without further data, the underlying question is what clinical guidelines say about the ideal side for peripheral venous cannulation in patients subjected to thoracoscopy [1-3]. The information on this is deceptively scarce, probably because it is considered a minor or futile issue for physicians (but not for some patients), which falls more under the guidelines for nurses or even anaesthesiologists than pulmonologists. 
A venous access is necessary for the administration of anaesthetics and sedatives or any anticipated need during pleural invasive procedures. On the rare occasions where a central venous catheter is considered, the catheter should be placed on the side of the thoracoscopy to prevent the eventuality of bilateral pneumothoraces. However, peripheral access is safer and easier to obtain than central access. As a general rule, the veins in the dorsum of the hand and, particularly, those of the non-dominant upper extremity, are preferred. Yet, in the case of thoracoscopy, the side of venous catheter placement is more closely related to the side of the procedure than handedness. The British Thoracic Society guidelines on local anaesthetic thoracoscopy state that an intravenous cannula should be placed in the hand on the same side as the planned procedure [4]. Conversely, a British book on thoracoscopy recommends a peripheral intravenous catheter insertion on the non-operative side [5]. Although other specialised books do not mention the ideal side for peripheral venous access as a patient preparation step for thoracoscopy, they show pictures indiscriminately using the ipsilateral and the contralateral sides to a certain hemithorax [6]. I have posed the question to 10 internationally renowned thoracoscopists, of whom six gave a uniform opinion; namely, to select the same side for peripheral venous cannulation as the hemithorax to be explored. This is because the patient is placed in a lateral decubitus position with the involved side up and the ipsilateral arm across or raised above the head, where it is easier to access for infusions of medications or fluids. However, the cuff bladder for blood pressure monitoring during thoracoscopy should not be applied to the arm used for peripheral venous access. The same reasoning is valid for inserting a chest drain if the chosen patient positioning is the lateral decubitus, but does not necessarily apply to other positions such as supine [7]. Notably, two experts believed that contralateral venous cannulation was preferable for anaesthesiologists, while the other two considered that the selected side for a venous access during thoracoscopy did not matter too much.

It is presumed that future guidelines on pneumothorax and pleural procedures will focus more on ambulatory management and patient-related outcome measures [8-10]. Among the latter, however, some apparently trivial aspects of clinical practice, as U. Kartoun observes, may need to be more clearly addressed.

@ERSpublications

For thoracoscopy, the side of venous catheter placement is often, but not always, related to the side of the procedure, but the effect on patient-related outcome measures should be addressed http://ow.ly/J03W30mrAxg

Cite this article as: Porcel JM. Improving the management of spontaneous pneumothorax. Eur Respir J 2018; 52: 1801918 [https://doi.org/10.1183/13993003.01918-2018].

José M. Porcel

Pleural Medicine Unit, Dept of Internal Medicine, Arnau de Vilanova University Hospital, IRBLleida, Lleida, Spain.

Correspondence: José M. Porcel, Pleural Medicine Unit, Dept of Internal Medicine, Arnau de Vilanova University Hospital, IRBLleida, Alcalde Rovira Roure 80, Lleida 25198, Spain. E-mail: jporcelp@yahoo.es

Received: Oct 062018 | Accepted: Oct 082018

Acknowledgements: Thanks to David Feller-Kopman (The Johns Hopkins University School of Medicine, Baltimore, MD, USA), Francisco Rodriguez-Panadero (Instituto de Biomedicina de Sevilla, Seville, Spain), Nick Maskell (University of Bristol, Bristol, UK), Marios Froudarakis (Medical School of Alexandroupolis, Alexandroupolis, Greece), Pyng Lee (National University of Singapore, Singapore), Gary Lee (Sir Charles Gairdner Hospital, Perth, Australia), Najib Rahman (Churchill Hospital, Oxford, UK), Philippe Astoul (Hospital North Aix-Marseille University, Marseille, France), Julius Janssen (Canisius Wilhelmina Hospital, Nijmegen, The Netherlands) and Peter Licht (Odense University Hospital, Odense, Denmark) for their helpful feedback.

Conflict of interest: J.M. Porcel has nothing to disclose.

\section{References}

1 MacDuff A, Arnold A, Harvey J. Management of spontaneous pneumothorax: British Thoracic Society Pleural Disease Guideline 2010. Thorax 2010; 65: Suppl. 2, ii18-ii31.

2 Rahman NM, Ali NJ, Brown G, et al. Local anaesthetic thoracoscopy: British Thoracic Society Pleural Disease Guideline 2010. Thorax 2010; 65: Suppl. 2, ii54-ii60.

3 Tschopp JM, Bintcliffe O, Astoul P, et al. ERS task force statement: diagnosis and treatment of primary spontaneous pneumothorax. Eur Respir J 2015; 46: 321-335.

4 Rahman NM, Ali NJ, Brown G, et al. Appendix 5. Local anaesthetic thoracoscopy. Practical guide to the procedure. London, British Thoracic Society, 2010. Available from: www.brit-thoracic.org.uk/standards-of-care/ guidelines/bts-pleural-disease-guideline/

5 Buchanan DR, Neville E, eds. Thoracoscopy for Physicians. A Practical Guide. London, Arnold, 2004 ; p. 72.

6 Lee P, Colt HF, eds. Flex-rigid Pleuroscopy. Singapore, CMPMedica, 2005; pp. 95-96. 
7 Porcel JM. Chest tube drainage of the pleural space: a concise review for pulmonologists. Tuberc Respir Dis 2018; 81: 106-115.

8 Thelle A, Gjerdevik M, SueChu M, et al. Randomised comparison of needle aspiration and chest tube drainage in spontaneous pneumothorax. Eur Respir J 2017; 49: 1601296.

9 Walker SP, Bibby AC, Halford P, et al. Recurrence rates in primary spontaneous pneumothorax: a systematic review and meta-analysis. Eur Respir J 2018; 52: 1800864.

10 Porcel JM. Phenotyping primary spontaneous pneumothorax. Eur Respir J 2018; 52: 1801455.

Copyright (C)ERS 2018 